\title{
Chaotic Adaptive Butterfly Mating Optimization and Its Applications in Synthesis and Structure Optimization of Antenna Arrays
}

\author{
Bin Li $\left(\mathbb{D},{ }^{1}\right.$ Chao Liu $\mathbb{D}^{1},{ }^{1}$ Huaning Wu $\left(\mathbb{D},{ }^{1}\right.$ Yifeng $\mathrm{Zhao}^{2}$ and Yinghui Dong ${ }^{1}$ \\ ${ }^{1}$ Department of Electronic Engineering, Naval University of Engineering, 430033, China \\ ${ }^{2}$ School of Information and Communication, National University of Defense Technology, 430000, China \\ Correspondence should be addressed to Chao Liu; liuchao0817@sina.com
}

Received 22 September 2018; Accepted 31 December 2018; Published 3 March 2019

Academic Editor: Sotirios K. Goudos

Copyright (C) 2019 Bin Li et al. This is an open access article distributed under the Creative Commons Attribution License, which permits unrestricted use, distribution, and reproduction in any medium, provided the original work is properly cited.

\begin{abstract}
A novel chaotic adaptive butterfly mating optimization (CABMO) is proposed to be used in synthesizing the beam pattern. In order to improve the optimization accuracy and avoid trapping in the local optimum, the homogeneous chaotic system and adaptive movement mechanism are combined into the proposed algorithm, where the initialization and redistribution of butterflies are chaotically dispersed with an adaptive movement closely related to the ultraviolet changes. After validating the performance of CABMO through several benchmark functions with different dimensions, the improved algorithm outperforms when compared to other state-of-the-art nature-inspired metaheuristic algorithms. The proposed algorithm is then used to understand any linear array problems in terms of the sidelobe reduction. Finally, a CABMO strategy is utilized to optimize the mutual coupling model of the closely spaced VLF umbrella arrays. Results show that the optimized structure has comfortably outperformed the original structure. Full scanning of wave positions is realized from 15 to $30 \mathrm{kHz}$. The synthesis patterns are close to the theoretical optimum. The optimized results of the radiation performance and synthesized patterns demonstrate that the pattern synthesis and antenna structure optimization based on the CABMO algorithm provides a novel idea for antenna array optimization.
\end{abstract}

\section{Introduction}

The capability of beamforming, which can be manipulated by the phase shifts of antenna arrays, is one of the key evaluations of antenna designs [1]. In the real world, many advanced communication systems, such as massive MIMO antennas and adaptive arrays, have been empowered by using beamforming technology [2-4]. The main design objective of beamforming technology is to improve communication quality, preventing a polluted wastage of power, especially in high-power antenna systems.

Optimization algorithms designed on array geometries have been popular in recent years because of the capability of the pattern synthesis and other specific problems that do not require considerable human intervention [5-9]. Singh and Salgotra [10] applied an enhanced flower pollination algorithm to a linear antenna array (LAA) for the pattern synthesis with sidelobe level (SLL) suppression and null control. Wu et al. [11] proposed a chaotic adaptive invasive weed optimization (CAIWO) to synthesize the planar nonuniform circular arrays and compared it with a genetic algorithm (GA) [12] and particle swarm optimization (PSO) [13, 14]. Panduro et al. [15] applied an evolutionary algorithm (EA) to design the steerable linear arrays. Scannable linear arrays are designed in reference [16] with amplitude and phase optimization for maximum sidelobe level reduction. The synthesis of an ultrawideband circular and sparse concentric ring array has been studied in references $[17,18]$. Mittal and Singh applied biogeography-based optimization (BBO) in reference [19] to design a Yagi-Uda antenna where gain and impedance are set as the optimization objective. Reference [20] used an improved fruit fly optimization algorithm to design a U-slot microstrip array antenna in order to achieve a higher impedance bandwidth. Huang et al. [21] 
combined least-square active element pattern expansion (LS-AEPE) and iterative Fourier transform. The patterns of two microstrip antenna arrays including mutual coupling are synthesized by the combined method. Liu et al. [22] proposed a more generalized version to synthesize unequally spaced arrays including mutual coupling. Sun et al. [23] utilized a hybrid approach to suppress the maximum SLL of a large concentric circular antenna array.

Among all intelligence algorithms, butterfly mating optimization (BMO) [24], inspired by the wise communication tools of heterosexual butterflies [25], has emerged as a new practical concept to solve optimization problems. BMO has been applied to thinning problems for a large antenna array [26] and matching network optimization [27] with characteristics of high practicality. BMO is a stochastic process with a fixed step length; hence, obtaining a false or slow convergence at a later time is unavoidable. In this paper, we propose a novel powerful variant of a BMO denoted by $\mathrm{CABMO}$ and investigate its attractive features. Adding an average chaos initialization and an adaptive updated engine improves the function of the BMO. Then, several benchmark functions are introduced to test the performance of the CABMO. In comparison with other powerful algorithms, such as CAIWO [11], GA [12], PSO [13], glowworm swarm optimization with changing step (CSGSO) [28], and standard $\mathrm{BMO}$, the search ability of this proposed algorithm has been verified. To further illustrate the superiority of the proposed algorithm, beam patterns of LAA with 10 and 16 elements are synthesized for maximum SLL minimization, respectively. Finally, we apply the CABMO for optimizing VLF umbrella antenna arrays with a mutual coupling suppression. The electromagnetic (EM) simulations are performed to evaluate the beamforming ability in practical conditions. The results show that the proposed approach achieves the best performance compared with other algorithms for the decoupling problem of VLF umbrella antenna arrays.

The rest of this paper is organized as follows. Section 2 briefly describes the basic theory of BMO and the modification of the proposed CABMO algorithm. Section 3 formulates performance analysis of the CABMO algorithm by testing the benchmark functions. In Section 4, a pattern synthesis formulation of the linear antenna arrays is discussed with multioptimized variables. In Section 5, the proposed CABMO is utilized in optimizing a practical VLF phased umbrella antenna array to obtain the maximum gains with the mutual coupling reduction. The conclusion is drawn in Section 6.

\section{The BMO Algorithm and Its Modification}

2.1. Description of BMO. The standard BMO algorithm was initially presented by Jada et al. in the literature [24]. BMO simulates the distributing behavior of ultraviolet (UV) and the mating process of butterflies. In the BMO approach, a certain number of butterflies are stochastically placed in the search space. The absorbed and updated UV of each butterfly dominates the election of local mate (l-mate) in each iterative process. 1-mate selection ensures that the best butterfly with the most UV with a distance quantization is saved. By attracting the healthiest 1-mate, all butterflies move to the optimal solution. The BMO algorithm can be summarized in 4 steps: (1) initialization, (2) UV distribution, (3) 1-mate selection, and (4) position update. Algorithm details can be found in the literature [24].

2.2. The Specialties and Shortcomings of the BMO. The l-mate selection scheme of the butterflies is in a core-like pattern for the BMO algorithm. All the butterflies in the space participate in the selection of the l-mate UV distribution, as described in Section 2.1. In patrolling, each individual has an equal right to absorb and reflect UV simultaneously in each iterative process. The 1-mate utilizes more UV searches in the search environment to distribute the $\mathrm{UV}$ at a mutual distance, improving the local convergence efficiency of the swarm algorithm. In contrast, most biomimetic algorithms would not allow the signal propagation in the whole space, such as the PSO. Another obvious advantage of $\mathrm{BMO}$ is that there is no additional consumption for individuals. Less predetermined parameters are required in the $\mathrm{BMO}$, unlike other methods such as CSGSO. CSGSO needs a volatilization factor, an updated rate of fluorescein, an updated rate of decision domain, and other parameters to fit different spaces that increase the parameter sensitivity and search consumption during the iterative process.

However, some shortcomings of the BMO limit the optimization capability of this algorithm. The most obvious one is the way that the individual butterfly is dispersed in the search zone. The production of a pseudorandom distribution generates a lack of individual diversification and unequal opportunity in early patrolling. As mentioned above, mutual distances are measured by the positions of butterflies, determining the exploitation capability. In initial iterations, a good dispersion will make the individual perceive the surroundings as much as possible. However, this discipline may fail in the uneven UV attraction. In some extreme cases, a butterfly near the border would be confused about the direction to the border as the UV increases with an increasing distance. Then, the intensification of the individuals is limited by an identical fixed step, which causes a lack of convergence precision in later iterations. A step size control mechanism is introduced by Yang et al. [27]. However, the step control is monotonous and sensitive to the parameters. It is not environment-related but manipulation-related, such as the iteration number, the harmonic index, and the range of the step size. Therefore, it is necessary for the BMO algorithm to balance the diversification in the whole space and exploration accuracy in the final solution.

2.3. Chaotic Adaptive Butterfly Mating Optimization. Aimed at the shortcomings above, a homogenous chaotic dispersion solution and adaptive movement mechanism are integrated into the BMO. The proposed CABMO algorithm is modified as follows:

2.3.1. Chaotic Dispersion Solution. A chaotic map exhibits a typical nonlinear ergodic behavior, which can generate 
random parameters without repetition according to its own law within a specific range of values. Therefore, the CABMO algorithm has a powerful ability to free the local optimum. Using a nonequiprobability distribution, many chaotic sequences are generated, such as a logistic map. We chose the homogeneous chaotic systems to enhance the initialization and dispersion of the BMO. This modified BMO is named Chaotic BMO (CBMO). The iterative model based on the homogenized chaotic system is expressed as follows [29]:

$$
\left.\begin{array}{l}
\mu(t+1)=-4.56 \mu(t)^{2}-0.56 \mu(t)+0.36, \\
X(t+1)=\frac{1}{\pi} \arcsin \left(\frac{57}{25} \mu(t+1)+\frac{7}{25}\right),
\end{array}\right\}
$$

where $\mu \in[-(1 / 2), 43 / 114]$ denotes the initialization sequence and $X \in[-(1 / 2), 1 / 2]$ denotes the chaotic search sequence. Figure 1 shows the distribution of the chaotic sequence $X$ in the definition domain of $\mu$ with 1000 iterative points. The chaotic distribution is humongous and stochastic in the definition domain. Unlike pseudorandom sequences, the chaotic sequence has the characteristics of ergodicity, which is neither periodicity nor convergence in the mapping process. It is sensitive to the values of initialization for the chaotic sequence. The linear complexity of the chaotic distribution is $N / 2$, which is much larger than that of pseudorandom sequences. Hence, when the diversity of the butterflies increases, the capability of global search is improved.

2.3.2. Adaptive Movement Mechanism. In the iteration process, the search range of the optimization variable is extended to the subregion between the current variable and the l-mate by introducing a UV-based factor. The movement update is represented as follows:

$$
\begin{aligned}
& P_{i}(t+1)=P_{i}(t)+\omega(t) \cdot\left(P_{\text {l-mate }}(t)-P_{i}(t)\right), \\
& \left\{\begin{array}{l}
\omega(t)=\exp \left(\frac{-\lambda(t)}{\lambda(t-1)+1}\right), \\
\lambda(t)=\frac{\mathrm{UV}_{i}(t)}{\mathrm{UV}_{i}(t-1)},
\end{array}\right.
\end{aligned}
$$

where $\omega(t)$ denotes the adaptive inertia factor for the $t$ th iterations and $P_{i}(t)$ and $P_{\text {l-mate }}(t)$ denote the position of the butterfly $i$ and l-mate in the $t$ th iteration, respectively. When the butterfly $i$ moves to its l-mate, the increment value of the $\mathrm{UV}$ is increased as the butterfly $i$ moves closer to the global optimum. The butterfly $i$ will increase pace and expand the search zone. In contrast, the butterfly $i$ will adaptively move closer to the l-mate and increase the global search ability as the UV increment value decreases.

2.3.3. $C A B M O$. The optimization objective is to minimize $f\left(X_{1}, X_{2}, \ldots, X_{d}\right)$ subject to $X_{\min }^{n}<X_{n}<X_{\max }^{n}$, where $n=$ $1,2, \ldots, d$ and $d$ denotes the dimension of the search space. $X=\left(X_{1}, X_{2}, \ldots, X_{d}\right)$ in practical problem represents

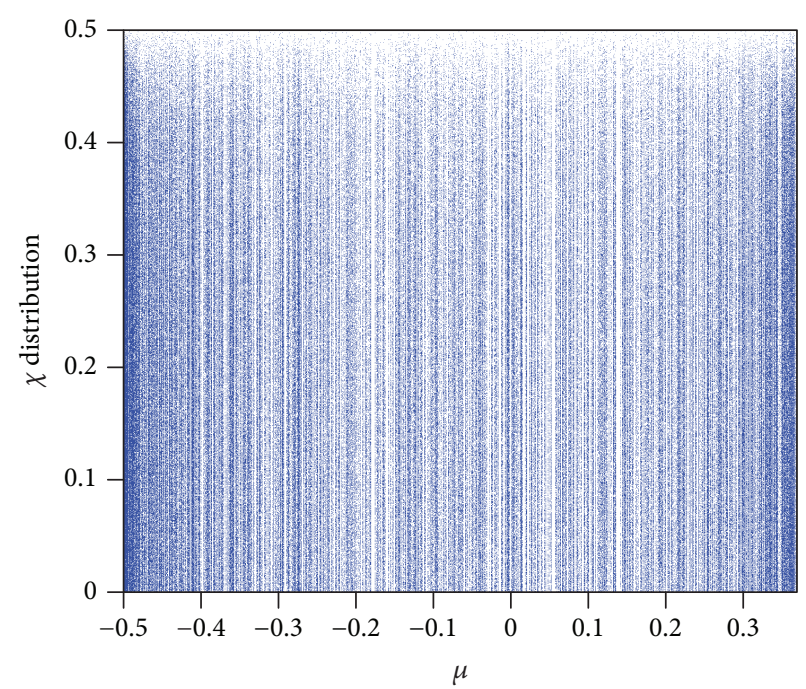

FIgURE 1: Distribution of the chaotic sequence $X$ over the variables in the definition domain with 1000 points.

a set of solutions. The proposed CABMO can be described as follows.

(1) Initialization. Candidate sequences are initialized in the definition domain of the homogenized chaotic system described in Section 2.3.1

(i) Transform variable $X$ to $\mu$ in the range of $(-(1 / 2), 43 / 114)$ :

$$
\mu=\frac{50\left(X-X_{\min }\right)}{57\left(X_{\max }-X_{\min }\right)}-\frac{1}{2} .
$$

(ii) Chaotically distribute $\mu$ to a novel $X^{\prime}$ using the homogenized chaotic system

(iii) Transform the chaotic variable $X^{\prime}$ to the search space in special optimization problem as follows:

$$
\bar{X}=\frac{X_{\max }+X_{\min }}{2+X^{\prime}\left(X_{\max }-X_{\min }\right)},
$$

where $X_{\max }$ and $X_{\min }$ stand for the maximum and minimum values of the spaces, respectively.

(2) UV Update. Evaluate the fitness of each individual in the iteration and update the UV values based on their fitness values

(3) l-mate Selection. Calculate the scatter values according to the distance between the butterflies, sort them, and select the l-mate

(4) Movement Update. Considering the position of the l-mate, the velocity and position of each butterfly are updated using equation (2). The adaptive inertia factor is calculated for each butterfly by 
TABLE 1: The results of benchmark functions.

\begin{tabular}{|c|c|c|c|c|c|}
\hline Benchmark function & Algorithm & Best result & Worst result & Average result (peak catching rate) & Standard deviation \\
\hline \multirow{7}{*}{$f_{1}(d=10)$} & PSO & $6.372 E-03$ & 1.952 & $5.334 E-01$ & $5.93 E-02$ \\
\hline & GA & $2.351 E-02$ & 2.293 & $7.941 E-01$ & $6.42 E-01$ \\
\hline & CSGSO & $1.273 E-02$ & $7.291 E-01$ & $2.812 E-02$ & $4.24 E-03$ \\
\hline & CAIWO & $4.283 E-05$ & $2.836 E-01$ & $3.772 E-03$ & $1.32 E-02$ \\
\hline & $\mathrm{BMO}$ & $4.972 E-02$ & 1.532 & $9.340 E-01$ & $2.93 E-02$ \\
\hline & СBMO & $9.732 E-03$ & $5.392 E-02$ & $1.035 E-02$ & $5.91 E-03$ \\
\hline & $\mathrm{CABMO}$ & $6.981 E-06$ & $8.735 E-05$ & $4.673 E-05$ & $4.20 E-04$ \\
\hline \multirow{7}{*}{$f_{2}(d=10)$} & PSO & $3.621 E-02$ & 2.317 & $1.231 E-01$ & $5.57 E-01$ \\
\hline & GA & $8.386 E-02$ & 2.681 & $1.382 E-01$ & $7.32 E-01$ \\
\hline & CSGSO & $5.382 E-04$ & $5.239 E-03$ & $3.431 E-03$ & $7.32 E-06$ \\
\hline & CAIWO & $3.823 E-06$ & $6.351 E-04$ & $8.372 E-05$ & $5.59 E-06$ \\
\hline & $\mathrm{BMO}$ & $7.936 E-03$ & 1.002 & $7.113 E-02$ & $5.38 E-02$ \\
\hline & $\mathrm{CBMO}$ & $7.173 E-03$ & 1.791 & $7.031 E-02$ & $9.42 E-02$ \\
\hline & CABMO & $4.816 E-08$ & $3.122 E-05$ & $4.193 E-06$ & $3.52 E-06$ \\
\hline \multirow{3}{*}{$f_{3}(d=4)$} & $\mathrm{BMO}$ & 4 & 2.158 & $64.2 \%$ & $2.44 E-02$ \\
\hline & CBMO & 4 & 2.973 & $92.2 \%$ & $1.58 E-02$ \\
\hline & CABMO & 4 & 2.989 & $94.6 \%$ & $3.57 E-04$ \\
\hline
\end{tabular}

equation (3). The convergence velocity is much faster because of the adaptive velocity changes

(5) Redistribution. Evaluate the position of each butterfly according to the optimization range of variable. If the butterfly is out of the border, redistribution is implemented by using a chaotic search

(6) Repeat step (2) to step (5) until an ending criterion or the maximum number of iterations is achieved

\section{Performance Analysis}

In order to verify the optimization performance of the CABMO algorithm, the typical benchmark functions are tested for comparison and optimization. The experimental environment of this study is Windows 7 OS with Intel Xeon ${ }^{\circledR}$ (2.2 GHz CPU and 128GB RAM). Writing of algorithm codes is performed in MATLAB 2014a.

3.1. The Benchmark Function Test. The benchmark functions have been calculated in different dimensions. Each function was simulated with the swarm size of 500 . Because of the randomness of the algorithms, the experiments are independently performed 30 times. The obtained results using the CABMO are compared with those using the PSO, GA, CSGSO, and CAIWO. The configuring parameters of the PSO [13] are globe factor $w_{\mathrm{g}}=1.49618$, self-factor $w_{\mathrm{s}}=$ 1.49618 , and inertia factor $w_{\mathrm{i}}=0.7298$. The GA parameters [12] are set as mutation probability $P_{\mathrm{m}}=0.05$ and crossover probability $P_{\mathrm{c}}=0.95$. The CSGSO parameters [28] are volatile factor $\rho=0.6$, fluorescein turnover rate $\gamma=0.6$, and update rate of the decision domain $\beta=0.08$. The size of the initial population $P_{0}=100$ and maximum population $P_{\max }=500$ is taken for the CAIWO [11], respectively, and for the maximum seed number, $S_{\max }$ is fixed to 4 .

Rastrigen function : $f_{1}\left(x_{i}\right)=10 d+\sum_{i=1}^{d}\left[x_{i}^{2}-10 \cos \left(2 \pi x_{i}\right)\right]$,

Griewank function : $f_{2}\left(x_{i}\right)=\sum_{i=1}^{d} \frac{x_{i}^{2}}{4000}-\prod_{i=1}^{d} \cos \left(\frac{x_{i}}{\sqrt{i}}\right)+1$,

Equal peak function : $f_{3}\left(x_{1}, x_{2}, \ldots, x_{d}\right)=\sum_{i=1}^{d} \cos ^{2}\left(x_{i}\right)$.

The optimization results such as the best result, worst result, average result, and standard deviation are presented in Table 1. As shown in Table 1 by the average results and standard deviation, the solution of the proposed CABMO algorithm is more accurate and steady than that of the other algorithms. By adding the mechanism of the adaptive movement, each individual makes a step change based on the UV update and becomes more purposeful under the guidance of the l-mate. This adaptive mechanism promotes the convergence speed and accuracy especially in the later iteration. According to the peak capture rate of the function $f_{3}$, the randomness of the homogeneous chaotic system intensifies the diversity of the butterflies, effectively enhancing the capability of global search. As expected, we can see that the CABMO with a lower average time has a faster convergence speed. Compared with the BMO algorithm, the computing consumption of the CABMO is mainly increased in 


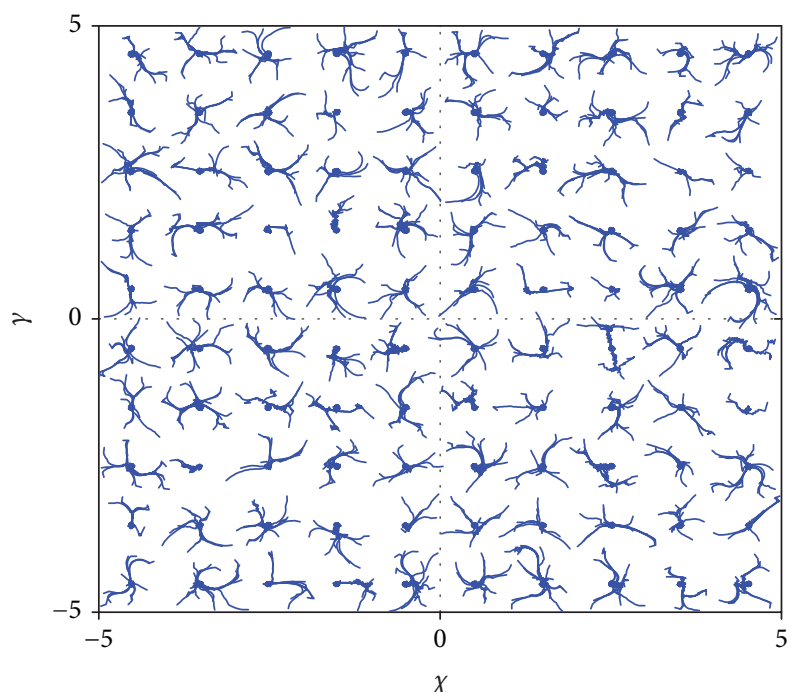

(a)

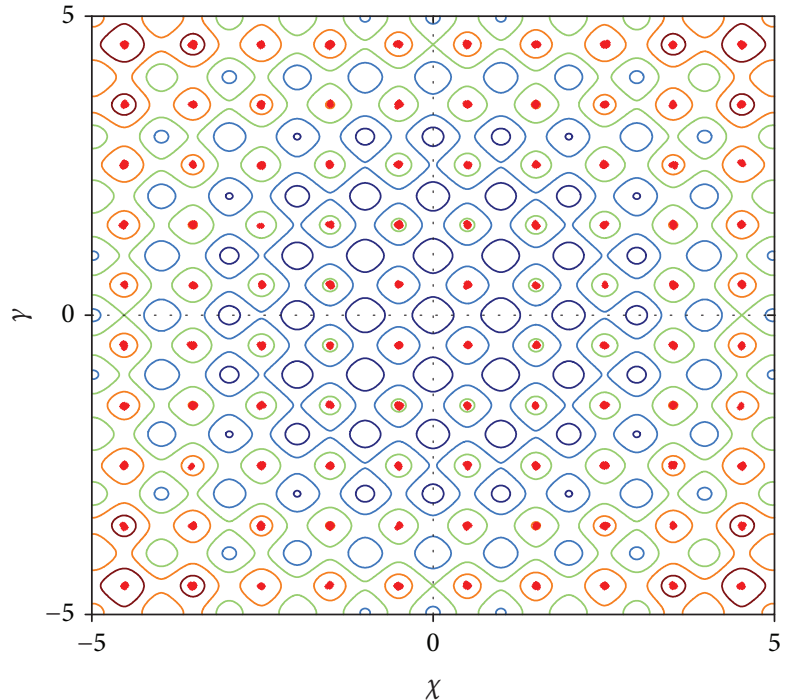

(b)

FIGURE 2: Iterative trajectories and the final butterfly distribution of the function $f_{1}$ : (a) iterative trajectories of butterflies by CABMO and (b) the final distribution of butterflies by CABMO.

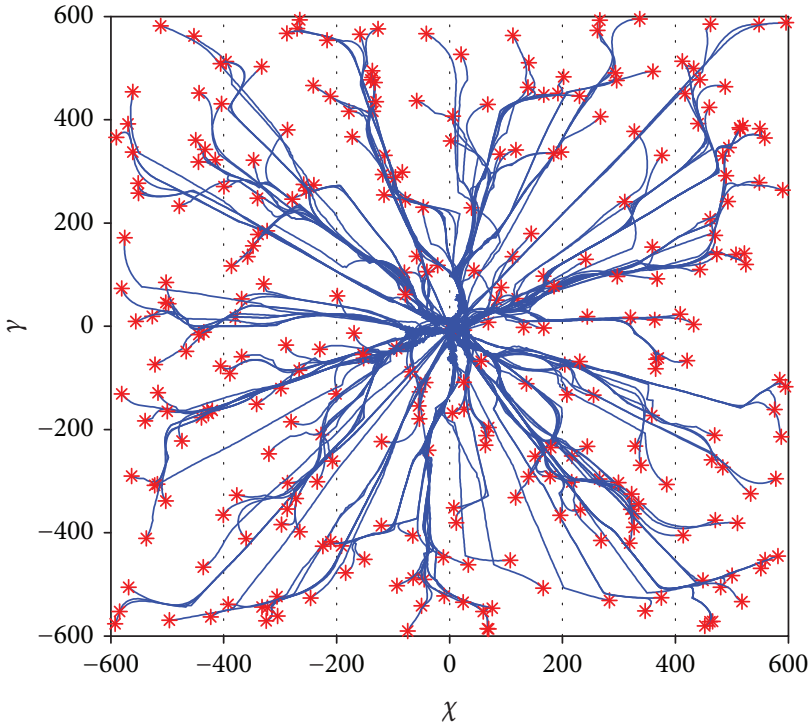

(a)

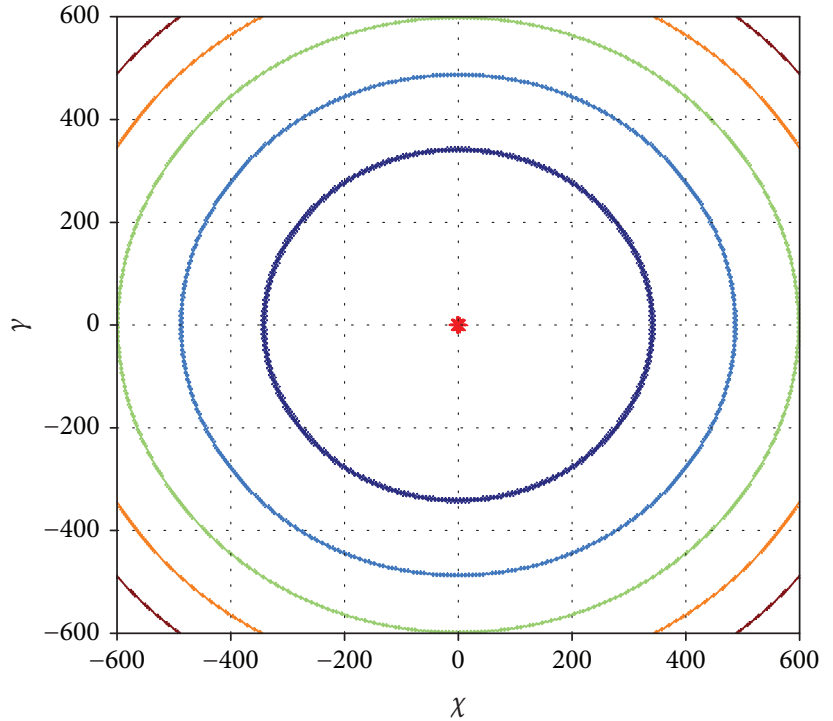

(b)

FIGURE 3: Iterative trajectories and the final distribution of butterflies of the function $f_{2}$ : (a) iterative trajectories of butterflies by CABMO and (b) the final distribution of butterflies by CABMO.

equation (4) and equation (5) chaotic initialization. However, the main factor of the proposed algorithm focuses on the calculation, sorting, and selection of the l-mate matrix in a standard BMO. According to the algorithm's progressive complexity theory, CABMO does not increase the complexity of a standard BMO.

As shown in Figure 2, the butterflies have been successfully captured by a maximum of 100 points when patrolling in the solution set of the function $f_{1}$. The iterative trajectories of butterflies by CABMO in Figure 2(a) clearly substantiate that the diversified distribution of the butterflies converges to the adjacent peak points without cross-region movement. Figure 2(b) shows the superior convergence precision of CABMO. The iterative trajectory and final distribution of the function $f_{2}$ are plotted in Figure 3. In Figure 3(a), we can see that in the early phase of the adaptive iteration, the individuals prefer to explore the search space globally. As the number of iterations increases, the adaptive factor changes the ability of the local optimization. There is no precocity of the butterflies in the optimization process of 


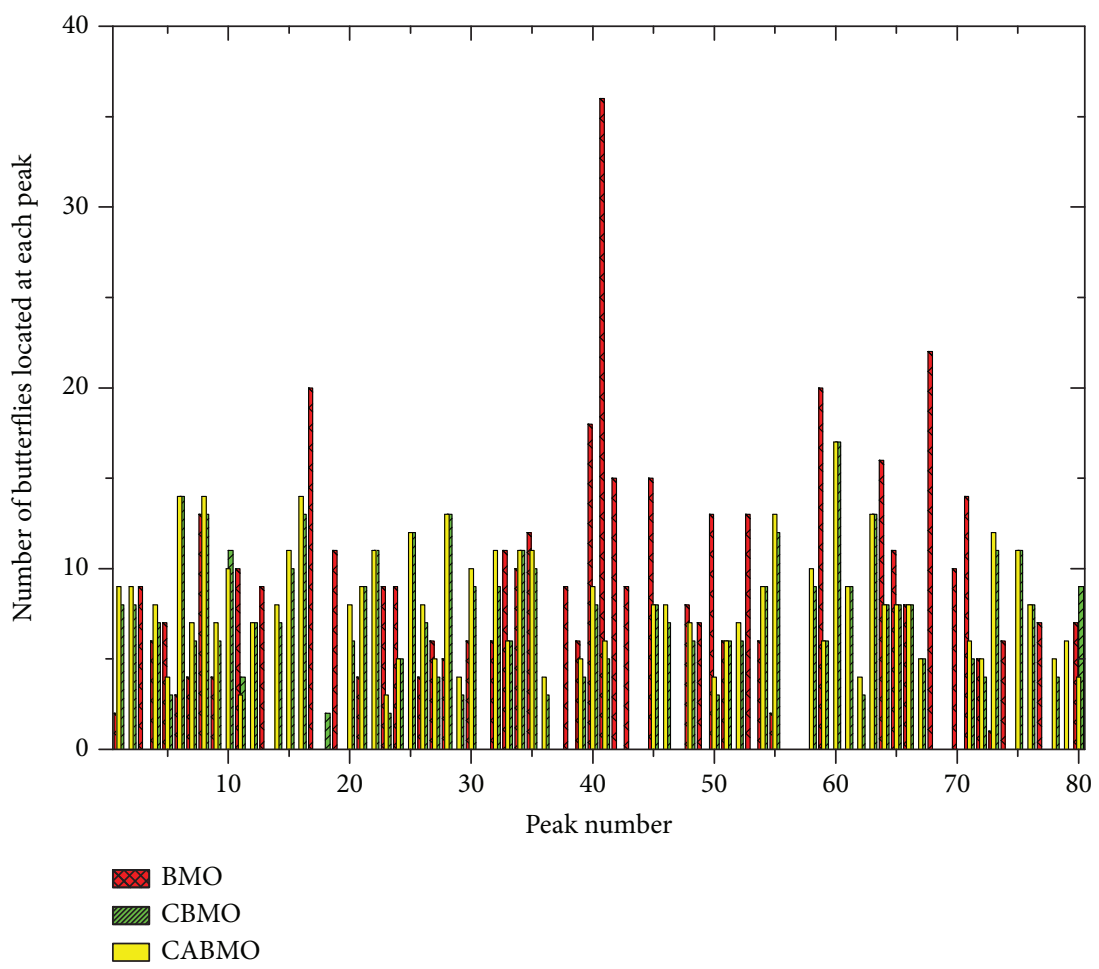

Figure 4: Number of butterflies located at each peak of the function $f_{3}$.

tending to the l-mate. Hence, the butterflies have a higher convergence precision and do not get trapped into local stagnations (Figure 3(b)). Figure 4 depicts the distribution number of butterflies located on the 81 maximal points of the function $f_{3}$. It is noteworthy that the points captured by the CABMO are more than those captured by the others. Due to the homogenous chaotic system, the diversification of the butterflies improves the global optimization ability during the early iteration. Then, redistribution of the individuals intensifies the vitality in the search space. Using the benchmark function test, the improved CABMO effectively balances the ability of global patrolling and local exploitation, especially in the multimodal and highdimensional functions.

\section{Linear Antenna Array Synthesis}

In this section, the CABMO approach is employed to synthesize the beamforming patterns of the LAAs for peak SLL minimization. The array factor (AF) for a LAA with $2 \mathrm{~N}$ elements placed symmetrically along the $x$-axis is expressed as follows [30]:

$$
\operatorname{AF}(\theta)=2 \sum_{n=1}^{N} I_{n} \cos \left(k x_{n} \cos (\theta)+\psi_{n}\right)
$$

where $I_{n}, x_{n}$, and $\psi_{n}$ denote the current amplitude, the location, and the phase of the $n$th element of array, respectively, $k$ denotes the wave number, and $\theta$ denotes the angle between the array and the positive $x$-axis. In order to obtain the peak SLL minimization, the fitness function is designed to synthesize the LAA as follows:

$$
\text { fitness }=\min _{\theta \in \theta_{\varepsilon}}(\max (20 \log |A F(\theta)|))
$$

where $\theta_{\varepsilon}$ denotes the special directivity for the minimization of SLL. To illustrate the effectiveness of the CABMO algorithm, three instantiations of the LAA are introduced for optimization of locations and current amplitudes, respectively.

4.1. Case One: Location Optimization of the 10-Element LAA. In the first example, the elements' locations of a LAA with the 10 elements are synthesized by different algorithms while maintaining uniform current amplitude and phase. The design goal for this example is to obtain minimum SLL in the regions, $\theta=\left[-90^{\circ},-16^{\circ}\right]$ and $\theta=\left[16^{\circ}, 90^{\circ}\right]$. The optimization processes are independently executed by 15 times. Using the proposed approach, the values of the optimized locations and the standard deviation of the maximum SLL are shown in Table 2 and the beam pattern is depicted in Figure 5. The maximum SLLs optimized by the proposed algorithm and PSO [31], ACO [32], CSO [33], and BMO are listed in Table 3.

In Figure 5, the beam pattern of the 10-element LAA obtained by CABMO has the lowest peak SLL among the various nature-inspired algorithms, which shows that the CABMO achieves excellent outperformance. It is noteworthy that the first null beam width (FNBW) is increased from $23^{\circ}$ to $40.12^{\circ}$ after the CABMO optimization. A lower 
TABLE 2: The optimized results of the 10-element LAA for case one.

\begin{tabular}{lcr}
\hline Algorithm & Optimized locations & Standard deviation \\
\hline BMO & $0.2190 \lambda, 0.3623 \lambda, 0.8072 \lambda, 1.0919 \lambda, 1.6836 \lambda$ & 1.0306 \\
CABMO & $0.1172 \lambda, 0.3763 \lambda, 0.7537 \lambda, 0.9946 \lambda, 1.5687 \lambda$ & 0.8647 \\
\hline
\end{tabular}

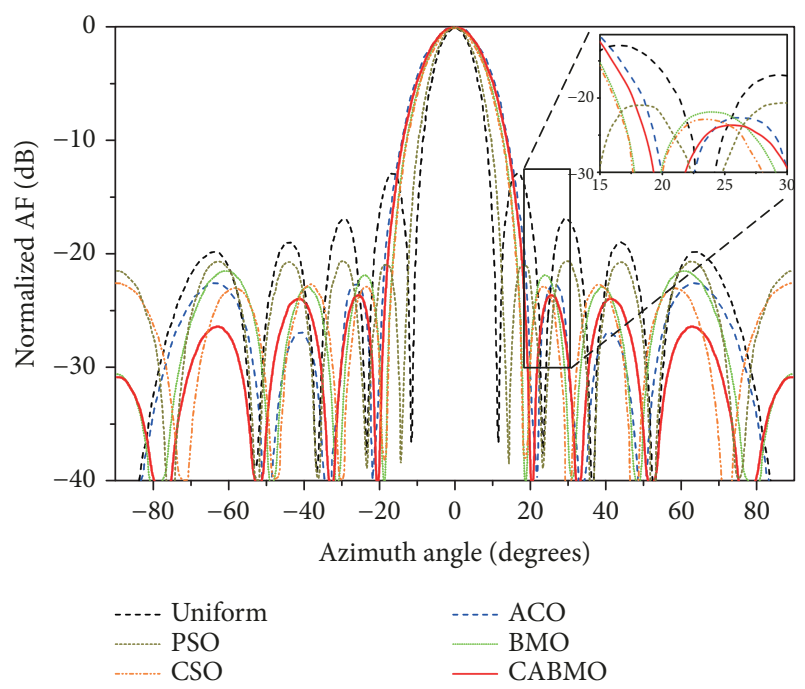

FIGURE 5: Nominalized AF of the 10-element LAA for case one.

TABLe 3: The maximum SLL for the 10-element LAA for case one.

\begin{tabular}{lc}
\hline Algorithm & Maximum SLL $(\mathrm{dB})$ \\
\hline Uniform & -12.97 \\
PSO [30] & -20.72 \\
ACO [31] & -22.66 \\
CSO [32] & -22.98 \\
BMO & -22.52 \\
CABMO & -23.65 \\
\hline
\end{tabular}

SLL is achieved at the expense of the FNBW. From Table 2, it is seen that CABMO has better accuracy performance than the BMO from the perspective of the standard deviation of the maximum SLL. In Table 3, it can be seen that the peak SLL obtained by BMO is $-22.52 \mathrm{~dB}$, which has been lowered from $-20.72 \mathrm{~dB}$ to $-22.52 \mathrm{~dB}$ as compared to the SLL of the LAA optimized by the PSO. The CABMO algorithm presents a maximum SLL of $-23.65 \mathrm{~dB}$, which is $10.68 \mathrm{~dB}$ lower than the peak SLL of the uniform array, which is lower by $2.93 \mathrm{~dB}, 0.99 \mathrm{~dB}$, $0.67 \mathrm{~dB}$, and $1.13 \mathrm{~dB}$ than the SLL of the PSO-, ACO-, CSO-, and BMO-optimized array, respectively.

4.2. Current Amplitude Optimization of the 16-Element LAA. The CABMO is used to synthesize the current amplitude of a 16-element LAA for SLL minimization. The relationship between AF and $I_{n}$ is nonlinear. The values of $x_{n}$ and $\psi_{n}$ are taken as $0.5 \lambda$ and 0 , respectively, in the AF equation (7).
4.2.1. Case Two. For this optimization case, the fitness function for this problem is mentioned in equation (8). The butterfly size is set as 20. The iteration number is 200 . The optimized current amplitudes obtained by BMO and CABMO are presented in Table 4. The peak SLLs optimized by different nature-inspired algorithms are shown in Table 5. The beam patterns of a 16-element LAA are shown in Figure 6. In addition, the parameters and results used for comparison are presented by reference [34].

In Figure 6, we can know that the normalized $\mathrm{AF}$ synthesized by CABMO algorithms have the lowest SLL (corresponding to the result of the 50 runs independently) for a 16-element LAA. As expected, we clearly show that CABMO is much better statistically than the other algorithms. From Table 4, it can be seen from the results that CABMO has a lower standard deviation of the peak SLLs than BMO. CABMO offers a maximum SLL of $-25.87 \mathrm{~dB}$, which is $12.72 \mathrm{~dB}$ lower as compared to the uniform array. As compared to PSO, BBO, FA, and BMO, the proposed method offers improvement of about $8.26 \mathrm{~dB}, 4.59 \mathrm{~dB}$, $1.60 \mathrm{~dB}$, and $4.62 \mathrm{~dB}$ in peak SLL (Table 5).

4.2.2. Case Three. The optimization objective for this example is to obtain minimum SLL in the regions, $\theta=\left[-90^{\circ},-16^{\circ}\right]$ and $\theta=\left[16^{\circ}, 90^{\circ}\right]$. For illustration of convergence depth, the butterfly size and iteration number are set as 40 and 1000 , respectively, which are larger than the configuring parameters in case two. The radiation patterns for this case are depicted in Figure 6. The optimized amplitudes of the BMO and CABMO arrays are illustrated in Table 6. The benchmark results are summarized in Table 7 .

In Figure 7 , it can be observed that the beam pattern optimized by the proposed algorithm (CABMO) offers the lowest maximum SLL among the compared algorithms. The results of standard deviation show that the performance of the proposed algorithm keeps robustness to initialization and is better than that of BMO (Table 6). From Table 7, the maximum SLL obtained by the CABMO algorithm is $-35.80 \mathrm{~dB}$, which is $22.65 \mathrm{~dB}$ lower than the SLL of the uniform array and $5.17 \mathrm{~dB}$ lower than the SLL of the PSO [14] array. The maximum SLL has been lowered from $-30.85 \mathrm{~dB}$ to $-35.80 \mathrm{~dB}$ (by $4.95 \mathrm{~dB}$ ) as compared to the SLL of the ant lion optimization- (ALO)- [31] optimized array.

\section{Closely Spaced Umbrella Antenna Arrays}

As the optimization problems of antenna arrays become more and more complex, many nonlinear problems are ascribed to optimization models of discrete and continuous variables, such as the pattern synthesis of CCAA in reference [23]. To illustrate the practicality of the CABMO algorithm in designing antenna arrays in the real world, the proposed 
TABLE 4: The optimized results of the 16-element LAA for case two.

\begin{tabular}{lcr}
\hline Algorithm & Optimized current amplitudes & Standard deviation \\
\hline BMO & $0.9011,0.7008,0.6233,0.6212,0.5637,0.5224,0.4996,0.3981$ & 1.3601 \\
CABMO & $0.8777,0.8567,0.8167,0.6962,0.5788,0.5649,0.3512,0.3591$ & 0.5347 \\
\hline
\end{tabular}

TABLE 5: The maximum SLL of the 16-element LAA for case two.

\begin{tabular}{lc}
\hline Algorithm & Maximum SLL $(\mathrm{dB})$ \\
\hline Uniform & -13.15 \\
PSO & -17.61 \\
BBO & -21.28 \\
FA & -24.27 \\
BMO & -21.25 \\
CABMO & -25.87 \\
\hline
\end{tabular}

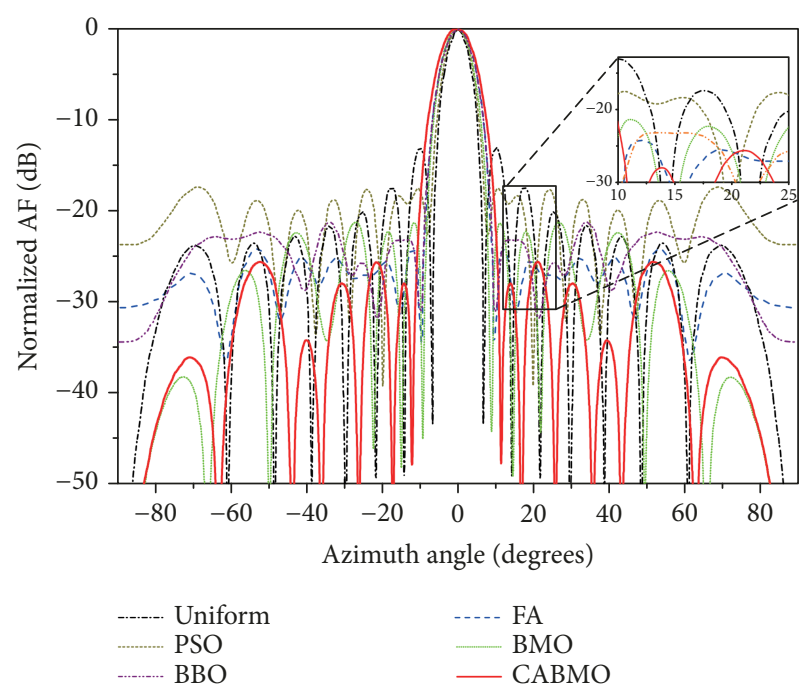

Figure 6: Nominalized AF of the 16-element LAA for case two.

algorithm will be applied to optimize the structure of the VLF umbrella antenna array introduced in reference [35] by suppressing the mutual coupling. The objective is to optimize the structure of the closely spaced arrays to intensify the array gains with a mutual coupling reduction. This optimization case is a combination of sparse and continuous optimization. Binary BMO (BBMO) [26] is applied to optimize the connection state of the down-lead wires. Further optimization of this model is obtained by the CABMO.

5.1. Mathematical Model of Antenna Arrays. Figure 8 shows the geometry model of the VLF umbrella antenna arrays, which can be regarded as monopole antenna arrays on a planar ground. The down-lead wires and the top wires are the main radiators. The antenna screen is composed of sling and multiple top wires. The coordinates of a single top wire satisfy a catenary equation. Under the conditions of both $\zeta=\zeta_{\max } / l<0.1$ and $h / l<0.1$, the catenary ab in Figure 9(a) can be approximated by the parabola equation. Selecting $N$ points $m_{1}, m_{2}, \ldots, m_{N}$ at equal intervals in the horizontal direction from the flat parabolic axis allows us to convert the points into a three-dimensional Cartesian coordinate system. The coordinates of these $N$ points are redefined as follows:

$$
\begin{aligned}
& \left\{\begin{array}{l}
x_{i}=x_{\mathrm{a}}-i \cdot \frac{x_{\mathrm{a}}-x_{\mathrm{b}}}{N}, \\
y_{i}=y_{\mathrm{a}}-i \cdot \frac{y_{\mathrm{a}}-y_{\mathrm{b}}}{N}, \\
z_{i}=h_{i}+l_{i} \cdot\left(x_{i}-n_{i}\right)^{2},
\end{array}\right. \\
& l_{i}=\frac{K}{l \cdot \tan \theta}, \\
& n_{i}=\frac{x_{\mathrm{a}}+x_{\mathrm{b}}-\left(z_{\mathrm{a}}-z_{\mathrm{b}}\right) /\left(l_{i} \cdot x_{\mathrm{a}}-l_{i} \cdot x_{\mathrm{b}}\right)}{2}, \\
& h_{i}=z_{\mathrm{a}}-l_{i} \cdot\left(x_{\mathrm{a}}-n_{i}\right)^{2},
\end{aligned}
$$

where $\left(x_{\mathrm{a}}, y_{\mathrm{a}}, z_{\mathrm{a}}\right)$ and $\left(x_{\mathrm{b}}, y_{\mathrm{b}}, z_{\mathrm{b}}\right)$ denote the coordinates of the fixed points a and $\mathrm{b}$, respectively, $i=1,2,3, \ldots, N$, $l \cdot \tan \theta=\sqrt{\left(x_{\mathrm{a}}-x_{\mathrm{b}}\right)^{2}+\left(y_{\mathrm{a}}-y_{\mathrm{b}}\right)^{2}+\left(z_{\mathrm{a}}-z_{\mathrm{b}}\right)^{2}}, \quad$ and $K>0$ denotes the sag coefficient. The average height of the top load can be represented as

$$
h_{\mathrm{t}}=\sum_{j=1}^{M} \sum_{i=1}^{N} \frac{z_{i j}}{M \cdot N}
$$

where $M$ represents the number of top wires in one piece of the antenna screen. Equations (9)-(11) show that as the endpoints of the top load are fixed, $K$ determines the sag and the average height of the antenna screen.

5.2. Problem Formulation. As mentioned above, this work is aimed at expanding the gain of the two-element phased arrays with a mutual coupling reduction. The input resistance of each array reflects the mutual coupling effect of the tightly coupled VLF vertical antennas with the interspacing $d_{12} \approx 1 / 8 \lambda$. Considering the antenna arrays as a two-port network, the input resistance of each array can be obtained as follows [35]:

$$
\left\{\begin{array}{l}
R_{1}=R_{11}+\operatorname{Re}\left(m e^{j \psi} Z_{12}\right), \\
R_{2}=R_{22}+\operatorname{Re}\left(\frac{1}{m} e^{-j \psi} Z_{21}\right),
\end{array}\right.
$$


TABLE 6: The optimized results of the 16-element LAA for case three.

\begin{tabular}{lcc}
\hline Algorithm & Optimized current amplitudes & Standard deviation \\
\hline BMO & $1.000,0.9325,0.8535,0.6989,0.5879,0.3888,0.3022,0.1991$ & 1.3067 \\
CABMO & $1.000,0.9308,0.8273,0.6603,0.5001,0.3210,0.2203,0.1219$ & 0.6538 \\
\hline
\end{tabular}

TABLE 7: The maximum SLL of the 16-element LAA for case three.

\begin{tabular}{lc}
\hline Algorithm & Maximum SLL (dB) \\
\hline Uniform & -13.15 \\
PSO [14] & -30.63 \\
ALO [30] & -30.85 \\
BMO & -31.88 \\
CABMO & -35.80 \\
\hline
\end{tabular}

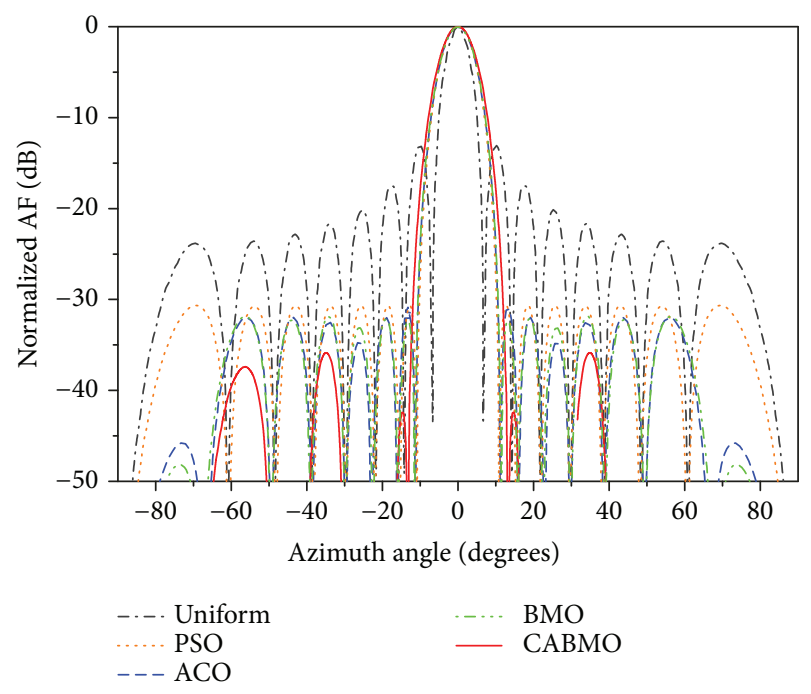

FIgURE 7: Nominalized AF of the 16-element LAA for case three.

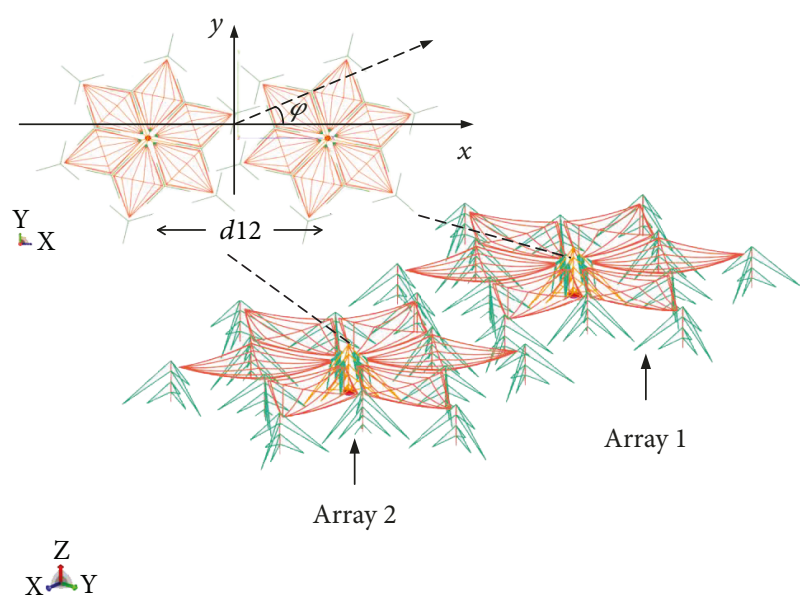

FIgURE 8: The model of the VLF umbrella antenna arrays.

$$
\begin{aligned}
& R_{22}=160 \pi^{2}\left(\frac{h_{\mathrm{e} 2}}{\lambda}\right)^{2} \\
& R_{21}=\frac{60 h_{\mathrm{e} 1} h_{\mathrm{e} 2}}{k d_{21}^{3}}\left[k d_{12} \cos \left(k d_{21}\right)-\left(1-k^{2} d_{21}^{2}\right) \sin \left(k d_{21}\right)\right],
\end{aligned}
$$

where $R_{11}$ and $R_{22}$ is, respectively, the mutual impedance of array 1 and array $2, \psi=-k d_{12} \cos \delta$ denotes the difference in the input current phase, $m=I_{2} / I_{1}$ denotes the ratio of the current amplitude, $h_{\mathrm{e} 1}$ and $h_{\mathrm{e} 2}$ are the effective height of array 1 and array 2, respectively, and $k$ denotes the wave number. Based on the transmission line theory, the equivalent height of the vertical antenna without the top load can be given as

$$
h_{0}=h_{\mathrm{t}}+h^{\prime}=\sum_{j=1}^{M} \sum_{i=1}^{N} \frac{z_{i}}{M \cdot N}+\frac{1}{k} c t g^{-1}\left(\frac{1}{Z_{0} \omega C_{h}}\right) \text {, }
$$

where $h^{\prime}$ is the equivalent antenna height contributed by the top load.

As shown in Figure 9(b), the down-lead wires of each array are synchronously excited by a power source. If the number of the radiation down-lead wires of array 1 is reduced, the current amplitude ratio $m$ will be reduced and correspond to the reduction area of the top load. Usually, the connection state, height, radius, and sag coefficient of the down-lead are all interacting parameters that influence $h_{\mathrm{el}}$ and $m$. Assuming the phase of array 2 is ahead of that of array 1, the optimization problem of the mutual coupling suppression for two-element phased arrays can be described as follows:

$$
\left.\begin{array}{rl}
\text { find } D_{\mathrm{opt}}=\left(d_{1}, d_{2}, \ldots, d_{n}\right)^{T}, h, r, \text { and } K_{i}, \\
d_{j}(j=1, \ldots, n) \in\{0,1\}, i=1, \ldots, m, \\
\max \quad G_{\mathrm{opt}} \\
\text { s.t. } \quad R_{1}, R_{2}>0 \\
\\
m_{i 2}>1, \\
& h_{\min } \leq h \leq h_{\max }, \\
& r_{\min } \leq r \leq r_{\max }, \\
0<K<K_{\mathrm{s}},
\end{array}\right\}
$$

where $h$ and $r$ are the height and radius of the down-lead wires, respectively, and $d_{i}(i=1, \ldots, n)$ is the connection state 


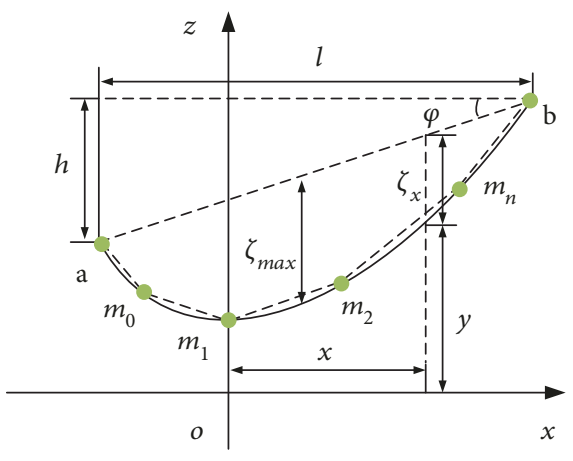

(a)

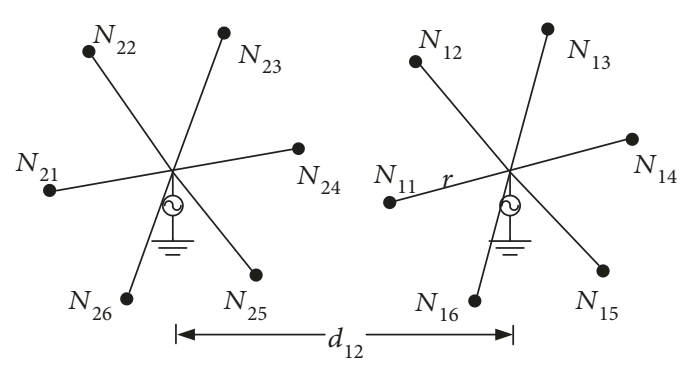

(b)

Figure 9: Schematics of the top wires and the down-lead wires. (a) The plane schematic of the top wires. (b) The topology diagram of down-lead wires.

of the $i$ th feeder. $d_{i}=0$ stands for the $i$ th grounded down-lead wire, and the corresponding feeder opens. $d_{i}=1$ stands for the $i$ th down-lead wire in regular work. $K_{\mathrm{s}}$ is the maximum sag coefficient. $G_{\text {opt }}$ is the array gain. $m_{i 2}$ is the current ratio between array $i$ and array 2. $h_{\min }$ and $h_{\max }$ are the lower and upper limits of the height of previous down-lead wires, respectively. $r_{\min }$ and $r_{\max }$ are the lower and upper limits of the radius of previous down-lead wires, respectively. The desired goal for this optimization case is to obtain the maximal array gains with a combination of sparse and continuous optimization. BBMO is applied to optimize the connection state of down-lead wires. The discrete position of the $i$ th butterfly in the BBMO approach is given as follows:

$$
\begin{aligned}
x_{i, k}(t) & = \begin{cases}\bmod \left(x_{i, k}(t-1), 2\right), & p \leq \xi(k), \\
x_{i, k}(t-1), & p \geq \xi(k),\end{cases} \\
p & =0.9 \times \frac{\operatorname{sum}\left(\bmod \left(x_{i}(t)+\mathrm{x}_{\text {l-mate }}(t), 2\right)\right)}{\sum_{j}\left(\operatorname{sum}\left(\bmod \left(\mathrm{x}_{j}(t)+\mathrm{x}_{\text {l-mate }}(t), 2\right)\right)\right)}+1,
\end{aligned}
$$

where $k$ and $t$ denote the dimension of the butterfly and iteration number, respectively, and $\xi(k)$ denotes the random parameter in the region of $[0,1]$. Further optimization of this model is obtained by CABMO. The fitness function can be transformed into a minimum optimization model as follows:

$$
f=\left|G_{\max }-G_{\text {opt }}\right|
$$

where $G_{\max }$ denotes the theoretical maximum gain. Considering the geometric symmetry of the two-element arrays, the beam positions are set as $\phi=0^{\circ}, 60^{\circ}, 90^{\circ}$. The optimization ranges of the variables $D_{\text {opt }}, h, r$, and $K$ are $\{0,1\},[220,240] \mathrm{m},[90,120] \mathrm{m}$, and $[0.1,0.5]$, respectively. The butterfly size and the number of iterations have been set to 20 and 200, respectively. In addition, a perfect electrical conductor plane is used for reducing the cost of
TABLE 8: List of the parameters of the antenna arrays.

\begin{tabular}{lc}
\hline Parameters & Values \\
\hline Operating frequency $(\mathrm{kHz})$ & 25 \\
Interspacing of the arrays $(\mathrm{m})$ & 1830 \\
Conductor conductivity $(\mathrm{S} / \mathrm{m})$ & $5.8 E+07$ \\
Relative permeability of the conductor & 1 \\
Horizontal scanning angles $\left(^{\circ}\right)$ & $0-360$ \\
The mesh length of the nonradiators $(\mathrm{m})$ & $\lambda / 200$ \\
The mesh length of the radiators $(\mathrm{m})$ & $\lambda / 300$ \\
\hline
\end{tabular}

the EM simulation. The simulation parameters of the antenna arrays are shown in Table 8.

5.3. Numerical Results and Discussion. As shown in Table 9, the optimization variables obtained by the CABMO satisfy the constraint conditions mentioned in Section 5.2. The array gains have been promoted by the structure optimization. The maximum gain of the optimized arrays in the end-fire direction reaches $1.16 \mathrm{~dB}$, which improves the radiation efficiency of the closely spaced array by $30.62 \mathrm{~dB}$ at $0^{\circ}$ beam position. To verify the performance of the CABMO algorithm, it is compared with the CAIWO [11] and CSGSO [28]. Figure 10 presents the normalized convergence curves when these 3 algorithms have been applied to the problem. CAIWO needs 120 iterations to reach the optimal values. The convergence time of the CSGSO up to the suboptimal value is 150 . As expected, high convergence accuracy and optimization efficiency are obtained by the CABMO algorithm. The convergence speed of the CABMO is the fastest, and the iteration reaches its best result when reaching 90 times around. The radiation resistance values of the optimization model are shown in Figure 11. It is noteworthy that the positive and negative signs of the longitudinal coordinates indicate the direction of the input current. Negative signs indicate that the current direction is from the highvoltage feeder to the tuning coil.

In Figure 11, the following conclusions can be drawn. First, within the same frequency, the phase-shifted feeding 
TABLE 9: The optimization variables obtained by the CABMO algorithm in $25 \mathrm{kHz}$.

\begin{tabular}{|c|c|c|c|c|c|c|}
\hline Algorithm & $\phi$ & $\begin{array}{c}D_{\text {opt }} \\
N_{21} N_{22} \ldots N_{26} N_{11} \ldots N_{16}\end{array}$ & $h(\mathrm{~m})$ & $r(\mathrm{~m})$ & K & Array gain $(\mathrm{dB})$ \\
\hline \multirow{3}{*}{ Nonoptimized } & $0^{\circ}$ & 111111111111 & 230.33 & 117.43 & 0.20 & - \\
\hline & $60^{\circ}$ & 111111111111 & 230.33 & 117.43 & 0.20 & 0.44 \\
\hline & $90^{\circ}$ & 111111111111 & 230.33 & 117.43 & 0.20 & 0.21 \\
\hline \multirow{3}{*}{ CABMO } & $0^{\circ}$ & 111011011111 & 230.33 & 117.43 & 0.27 & 1.16 \\
\hline & $60^{\circ}$ & 111111011111 & 236.58 & 101.92 & 0.21 & 0.53 \\
\hline & $90^{\circ}$ & 111111111111 & 239.45 & 97.69 & 0.16 & 0.29 \\
\hline
\end{tabular}

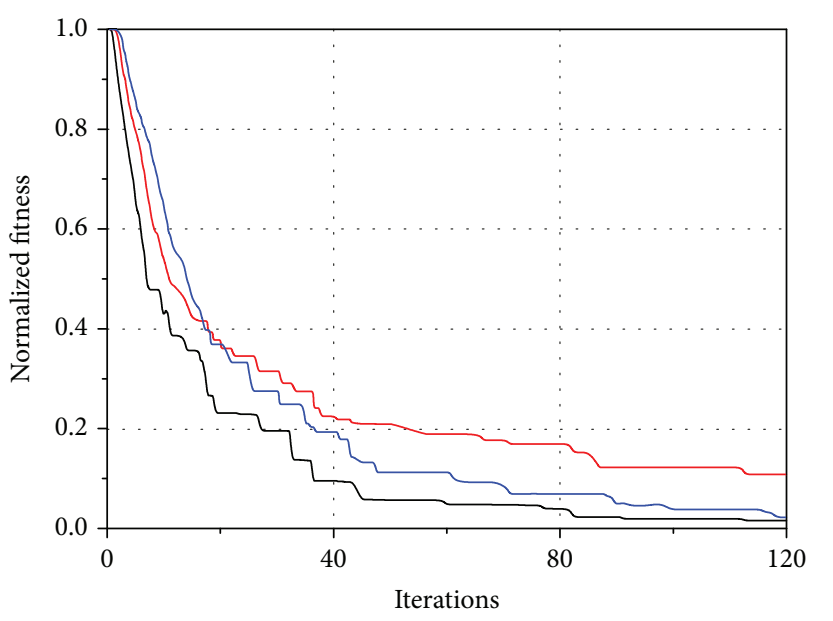

- CAIWO
CSGSO
CABMO

Figure 10: Convergence curves of three algorithms of the model in $25 \mathrm{kHz}$.

has a greater effect on the radiation resistances of the antenna arrays than the in-phase feeding. Figures 11(a) and 11(b) clearly show that the radiation resistances in the original structure are considerably influenced by the mutual coupling between the elements at $0^{\circ}\left(180^{\circ}\right)$ and $60^{\circ}\left(120^{\circ}\right)$ wave positions, respectively. The radiation resistance increases with the growth of the frequencies and is less than $1 \Omega$. We assume that electromagnetic waves cannot be effectively radiated in most parts of the VLF band, which verifies the necessity of optimization in this problem. After optimization, the radiation resistances of array 2 at $0^{\circ}\left(180^{\circ}\right)$ and $60^{\circ}\left(120^{\circ}\right)$ wave positions are improved obviously. At the $90^{\circ}\left(270^{\circ}\right)$ beam position, the radiation resistances do not decrease obviously by the thinning of the down-lead wires (Figure 11(c)). The beam combination in the new structure can be achieved at $15-30 \mathrm{kHz}$. The effectiveness of the proposed method is proven.

To further verify the performance of beamforming obtained by the CABMO algorithm, Figure 12 depicts the horizontal patterns of the optimized arrays at $25 \mathrm{kHz}$. In contrast, the theoretical optimal patterns without the consideration of the mutual coupling are also presented. In
Figure 12(a), the maximum gain of the arrays reaches $5.93 \mathrm{~dB}$ in the end-fire direction, which is $0.2 \mathrm{~dB}$ lower than the array gain of the original antenna arrays. The front-to-back ratios of the patterns reach $4.2 \mathrm{~dB}$. In Figure 12(b), due to the close interelement spacing, the horizontal pattern lobe of the closely spaced arrays is wide. Figure 12(c) illustrates that the optimization approach has little effect on the side direction pattern when the in-phase feeding is used. When the threshold of GRADE/SPREAD [36] is set as $85 \%$, the GRADE and SPREAD values of the global difference measure are 4 and 1 , respectively. The consistency between the optimized and theoretical optimal patterns verifies the correctness of the CABMO algorithm for the closely spaced arrays.

\section{Conclusions}

In this paper, a modified $\mathrm{BMO}$, entitled CABMO, is introduced and applied to synthesize linear and umbrella antenna arrays. The main advantage of the proposed algorithm over the state-of-the-art metaheuristic algorithms is that the CABMO has a powerful versatility to optimize different kinds of antenna arrays with an adaptive movement mechanism. The UV-based adaptive factor promotes exploitation as the search space changes. A homogeneous chaotic search assists the global exploration and fills a major gap in dispersion and redistribution of the butterflies. The performance analysis from several benchmark functions is presented in different dimensions. In addition, numerical results of LAA synthesis problems demonstrate the superiority of the improved algorithm to the other nature-inspired algorithms. Optimized antenna structures of the closely spaced umbrella antenna arrays were obtained using the CABMO for further suppression of mutual coupling. Taking the array gain as the fitness function, a nonlinear optimization model is established. The optimal results are compared with the arrays in the nonoptimized structure and with arrays produced by the other algorithms. The results verify the feasibility of the CABMO to be applied in the electromagnetics and antenna field for antenna array optimization. Future work in this area will include synthesizing other array geometries, further minimizing the maximum SLL of the LAA with FNBW constraint, and applying the decoupling method to other arrays. 


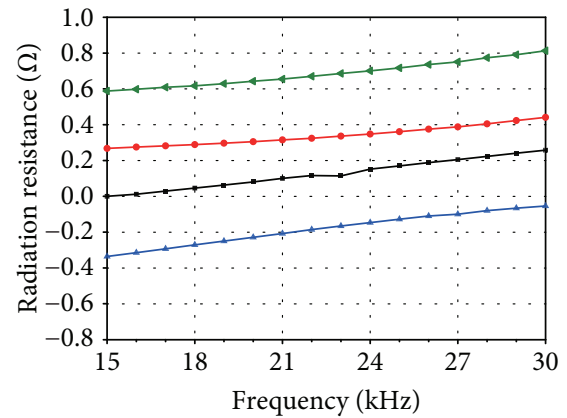

$\rightarrow$ Optimized structure, array 1

$\because$ Original structure, array 1

$\rightarrow$ Original structure, array 2

$\rightarrow$ Optimized structure, array 2

(a)

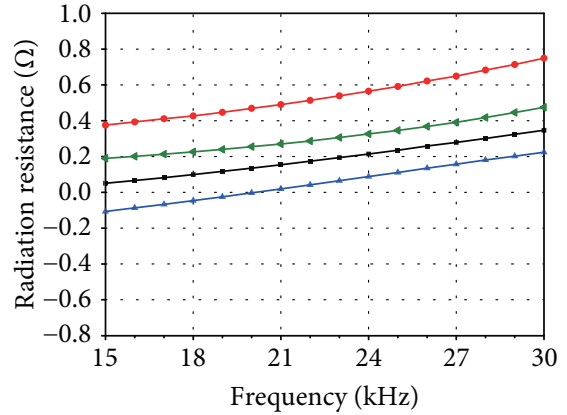

$\rightarrow$ Original structure, array 1

$\leftarrow$ Optimized structure, array 1

$\rightarrow$ Original structure, array 2

$\rightarrow$ Optimized structure, array 2

(b)

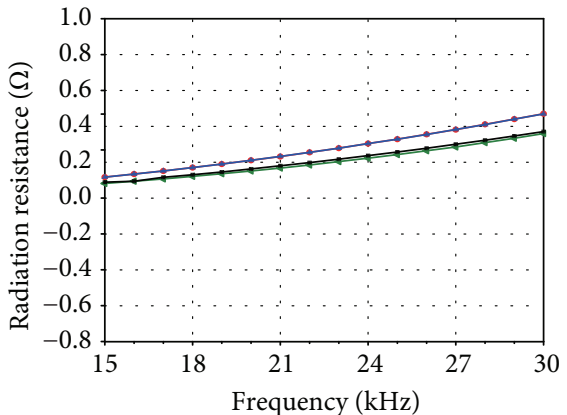

$\rightarrow$ Original structure, array 1

$\multimap$ Optimized structure, array 1

- Original structure, array 2

- Optimized structure, array 2

(c)

Figure 11: Radiation resistances of the VLF umbrella arrays for different beam positions: (a) $0^{\circ}\left(180^{\circ}\right)$ beam position; (b) $60^{\circ}\left(120^{\circ}\right)$ beam position; (c) $90^{\circ}\left(270^{\circ}\right)$ beam position.

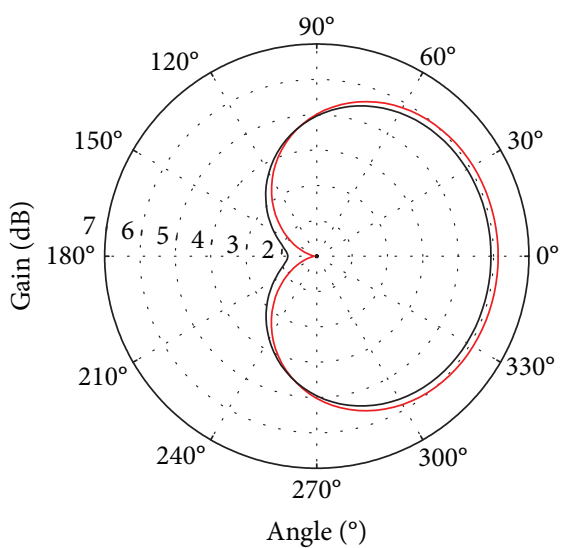

— Theoretical optimal structure — Optimized structure

(a)

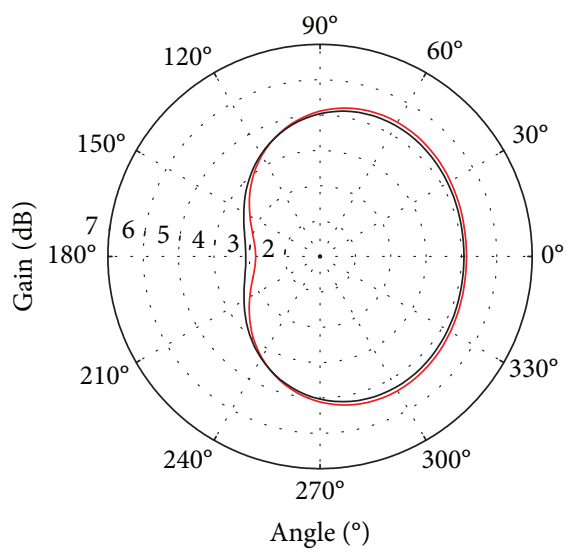

— Theoretical optimal structure — Optimized structure

(b)

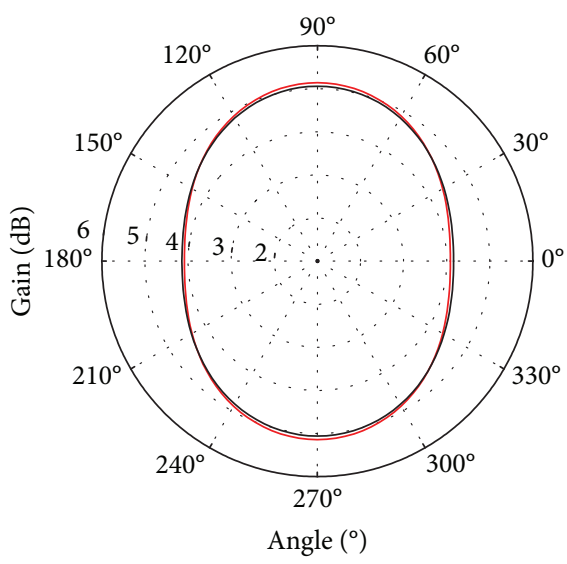

— Theoretical optimal structure — Optimized structure

(c)

FIgURe 12: Horizontal patterns of the VLF umbrella arrays by CABMO: (a) $0^{\circ}\left(180^{\circ}\right.$ ) beam position; (b) $60^{\circ}$ (120 ) beam position; (c) $90^{\circ}\left(270^{\circ}\right)$ beam position.

\section{Data Availability}

The data used to support the findings of this study are available from the corresponding author upon request.

\section{Conflicts of Interest}

The authors declare that there are no conflicts of interest regarding the publication of this paper.

\section{Acknowledgments}

This work was supported by the National Natural Science Foundation of China (Grant nos. 61302099 and 41504029) and Natural Science Foundation of Hubei Province (Grant no. 2015CFB174).

\section{References}

[1] H. R. E.-H. Bouchekara, A. Orlandi, M. Al-Qdah, and F. de Paulis, "Most valuable player algorithm for circular antenna arrays optimization to maximum sidelobe levels reduction," IEEE Transactions on Electromagnetic Compatibility, vol. 60, no. 6, pp. 1655-1661, 2018.

[2] M. Li, Y. Liu, and Y. J. Guo, "Shaped power pattern synthesis of a linear dipole array by element rotation and phase optimization using dynamic differential evolution," IEEE Antennas and Wireless Propagation Letters, vol. 17, no. 4, pp. 697-701, 2018.

[3] P. Chakravorty and D. Mandal, "Radiation pattern correction in mutually coupled antenna arrays using parametric assimilation technique," IEEE Transactions on Antennas and Propagation, vol. 64, no. 9, pp. 4092-4095, 2016.

[4] X. Wang, M. Amin, X. Wang, and X. Cao, "Sparse array quiescent beamformer design combining adaptive and 
deterministic constraints," IEEE Transactions on Antennas and Propagation, vol. 65, no. 11, pp. 5808-5818, 2017.

[5] Z. D. Zaharis, P. I. Lazaridis, J. Cosmas, C. Skeberis, and T. D. Xenos, "Synthesis of a near-optimal high-gain antenna array with main lobe tilting and null filling using Taguchi initialized invasive weed optimization," IEEE Transactions on Broadcasting, vol. 60, no. 1, pp. 120-127, 2014.

[6] D. Pinchera, M. D. Migliore, and G. Panariello, "Synthesis of large sparse arrays using IDEA (Inflating-Deflating Exploration Algorithm)," IEEE Transactions on Antennas and Propagation, vol. 66, no. 9, pp. 4658-4668, 2018.

[7] Z. Bayraktar, D. H. Werner, and P. L. Werner, "Miniature meander-line dipole antenna arrays, designed via an orthogonalarray-initialized hybrid particle-swarm optimizer," IEEE Antennas and Propagation Magazine, vol. 53, no. 3, pp. 4259, 2011.

[8] S. Jayaprakasam, S. K. Abdul Rahim, C. Y. Leow, T. O. Ting, and A. A. Eteng, "Multiobjective beampattern optimization in collaborative beamforming via NSGA-II with selective distance," IEEE Transactions on Antennas and Propagation, vol. 65 , no. 5, pp. 2348-2357, 2017.

[9] H. E. A. Laue and W. P. du Plessis, "Numerical optimization of compressive array feed networks," IEEE Transactions on Antennas and Propagation, vol. 66, no. 7, pp. 3432-3440, 2018.

[10] U. Singh and R. Salgotra, "Pattern synthesis of linear antenna arrays using enhanced flower pollination algorithm," International Journal of Antennas and Propagation, vol. 2017, Article ID 7158752, 11 pages, 2017.

[11] H. Wu, C. Liu, and X. Xie, "Pattern synthesis of planar nonuniform circular antenna arrays using a chaotic adaptive invasive weed optimization algorithm," Mathematical Problems in Engineering, vol. 2014, Article ID 575860, 13 pages, 2014.

[12] M. A. Panduro, A. L. Mendez, R. Dominguez, and G. Romero, "Design of non-uniform circular antenna arrays for side lobe reduction using the method of genetic algorithms," AEU International Journal of Electronics and Communications, vol. 60, no. 10, pp. 713-717, 2006.

[13] M. Shihab, Y. Najjar, N. Dib, and M. Khodier, "Design of non-uniform circular antenna arrays using particle swarm optimization," Journal of Electrical Engineering, vol. 59, no. 4, pp. 216-220, 2008.

[14] M. M. Khodier and M. Al-Aqeel, "Linear and circular array optimization: a study using particle swarm intelligence," Progress in Electromagnetics Research B, vol. 15, pp. 347-373, 2009.

[15] M. A. Panduro, C. A. Brizuela, and D. H. Covarrubias, "Design of electronically steerable linear arrays with evolutionary algorithms," Applied Soft Computing, vol. 8, no. 1, pp. 46-54, 2008.

[16] M. A. Panduro, A. Reyna, and J. Camacho, "Design of scannable linear arrays with amplitude and phase optimisation for maximum side lobe level reduction," International Journal of Electronics, vol. 96, no. 3, pp. 323-329, 2009.

[17] L. A. Garza, M. A. Panduro, D. H. Covarrubias, and A. Reyna, "Multiobjective synthesis of steerable UWB circular antenna array considering energy patterns," International Journal of Antennas and Propagation, vol. 2015, Article ID 789094, 9 pages, 2015.

[18] M. Ibarra, M. A. Panduro, A. G. Andrade, and A. Reyna, "Design of sparse concentric rings array for LEO satellites," Journal of Electromagnetic Waves and Applications, vol. 29, no. 15, pp. 1983-2001, 2015.
[19] E. Mittal and S. Singh, "Multiobjective gain-impedance optimization of Yagi-Uda antenna design using different BBO migration variants," Applied Artificial Intelligence, vol. 29, no. 1, pp. 33-48, 2015.

[20] A. Darvish and A. Ebrahimzadeh, "Improved fruit-fly optimization algorithm and its applications in antenna arrays synthesis," IEEE Transactions on Antennas and Propagation, vol. 66, no. 4, pp. 1756-1766, 2018.

[21] X. Huang, Y. Liu, P. You, M. Zhang, and Q. H. Liu, "Fast linear array synthesis including coupling effects utilizing iterative FFT via least-square active element pattern expansion," IEEE Antennas and Wireless Propagation Letters, vol. 16, pp. 804807, 2016.

[22] Y. Liu, X. Huang, K. D. Xu, Z. Song, S. Yang, and Q. H. Liu, "Pattern synthesis of unequally spaced linear arrays including mutual coupling using iterative FFT via virtual active element pattern expansion," IEEE Transactions on Antennas and Propagation, vol. 65, no. 8, pp. 3950-3958, 2017.

[23] G. Sun, Y. Liu, Z. Chen, S. Liang, A. Wang, and Y. Zhang, "Radiation beam pattern synthesis of concentric circular antenna arrays using hybrid approach based on cuckoo search," IEEE Transactions on Antennas and Propagation, vol. 66, no. 9, pp. 4563-4576, 2018.

[24] C. Jada, A. K. Vadathya, A. Shaik, S. Charugundla, P. R. Ravula, and K. K. Rachavarapu, "Butterfly mating optimization," in Intelligent Systems Technologies and Applications, S. Berretti, S. Thampi, and P. Srivastava, Eds., pp. 3-15, Springer, 2016.

[25] S. Ch, A. Shaik, C. Jada, and A. K. Vadathya, "Butterfly communication strategies: a prospect for soft-computing techniques," in 2014 International Joint Conference on Neural Networks (IJCNN), pp. 424-431, Beijing, China, July 2014.

[26] H. Wu, C. Liu, B. Li, and X. Xie, "A novel binary butterfly mating optimization algorithm with subarray strategy for thinning of large antenna array," Progress in Electromagnetics Research M, vol. 60, pp. 101-110, 2017.

[27] Y. L. Yang, C. Liu, and H. N. Wu, "An improved adaptive-step butterfly mating optimization algorithm," in 2017 3rd IEEE International Conference on Computer and Communications (ICCC), pp. 717-723, Chengdu, China, December 2017.

[28] Y.Z. Ou and Y. Q. Zhou, "Self-adaptive step glowworm swarm optimization algorithm," Journal of Computer Applications, vol. 31, no. 7, pp. 1804-1807, 2011.

[29] H. Y. Zang and H. F. Huang, "Research on algorithm of generating S-box based on uniform chaotic system," Journal of Electronics and Information Technology, vol. 39, no. 3, pp. 575-581, 2017.

[30] P. Saxena and A. Kothari, "Ant lion optimization algorithm to control side lobe level and null depths in linear antenna arrays," AEU - International Journal of Electronics and Communications, vol. 70, no. 9, pp. 1339-1349, 2016.

[31] L. Pappula and D. Ghosh, "Linear antenna array synthesis using cat swarm optimization," AEU - International Journal of Electronics and Communications, vol. 68, no. 6, pp. 540549, 2014.

[32] E. Rajo-Iglesias and O. Quevedo-Teruel, "Linear array synthesis using an ant-colony-optimization-based algorithm," IEEE Antennas and Propagation Magazine, vol. 49, no. 2, pp. 7079, 2007.

[33] P. Saxena and A. Kothari, "Optimal pattern synthesis of linear antenna array using grey wolf optimization algorithm," 
International Journal of Antennas and Propagation, vol. 2016, Article ID 1205970, 11 pages, 2016.

[34] G. Sun, Y. Liu, H. Li, S. Liang, A. Wang, and B. Li, “An antenna array sidelobe level reduction approach through invasive weed optimization," International Journal of Antennas and Propagation, vol. 2018, Article ID 4867851, 16 pages, 2018.

[35] B. Li, C. Liu, and Y. H. Dong, "Spatial power combining of VLF umbrella antenna arrays with multi-delay lines," Progress in Electromagnetics Research C, vol. 80, pp. 79-87, 2018.

[36] A. P. Duffy, A. Orlandi, and G. Zhang, "Notice of retraction: review of the feature selective validation method (FSV). Part I-theory," IEEE Transactions on Electromagnetic Compatibility, vol. 60, no. 4, pp. 814-821, 2018. 


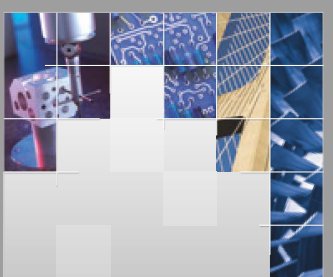

\section{Enfincering}
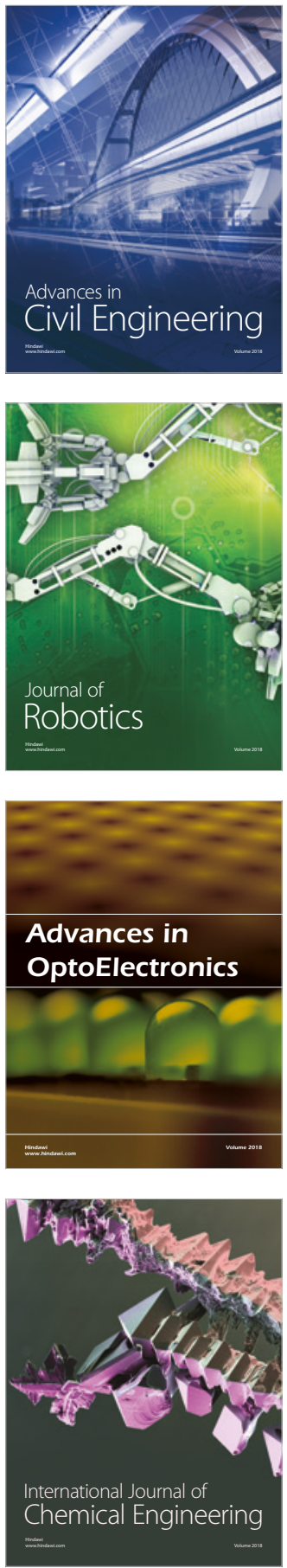

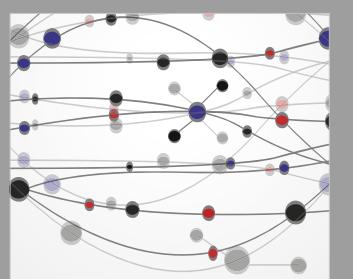

\section{Rotating \\ Machinery}

The Scientific World Journal

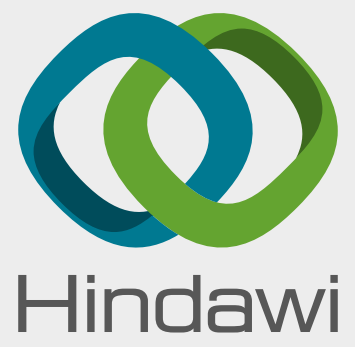

Submit your manuscripts at

www.hindawi.com
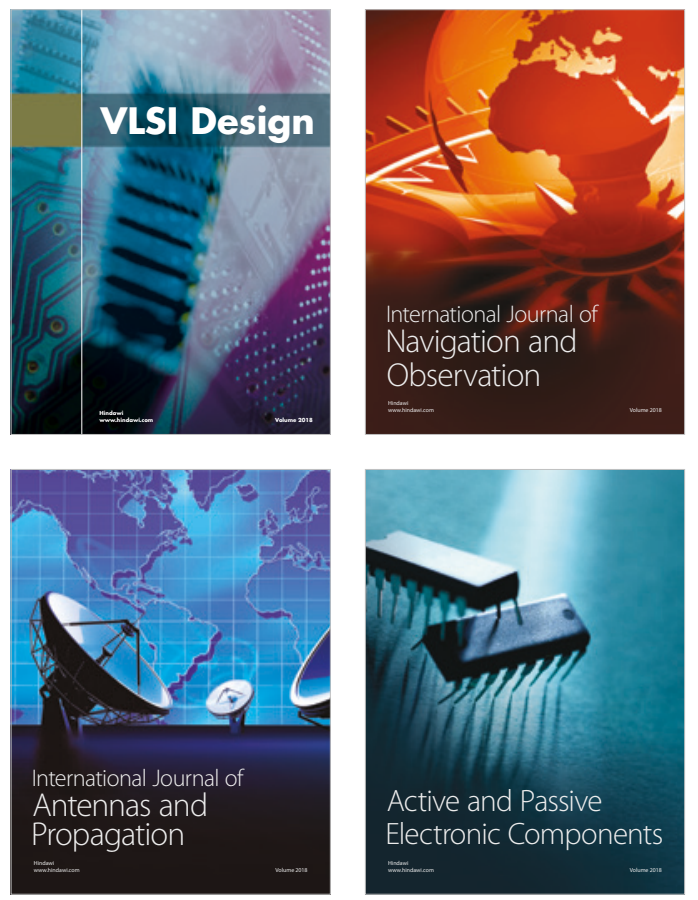
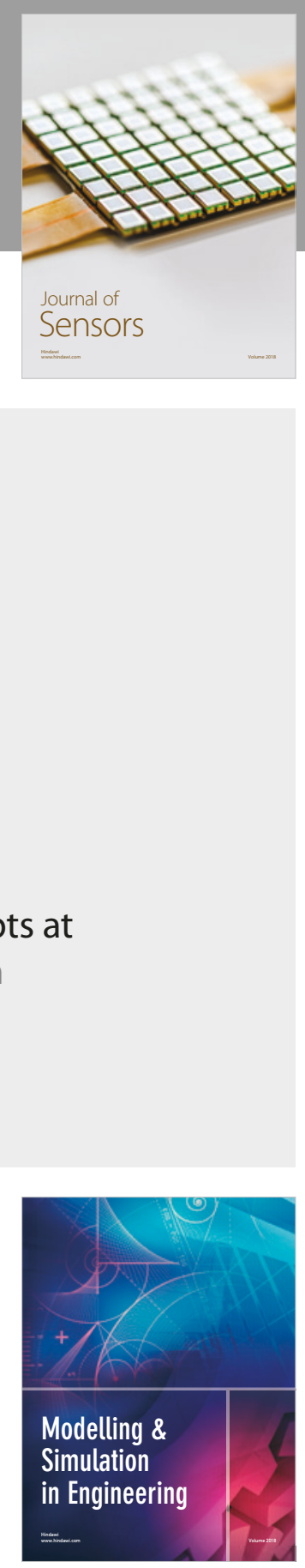

\section{Advances \\ Multimedia}
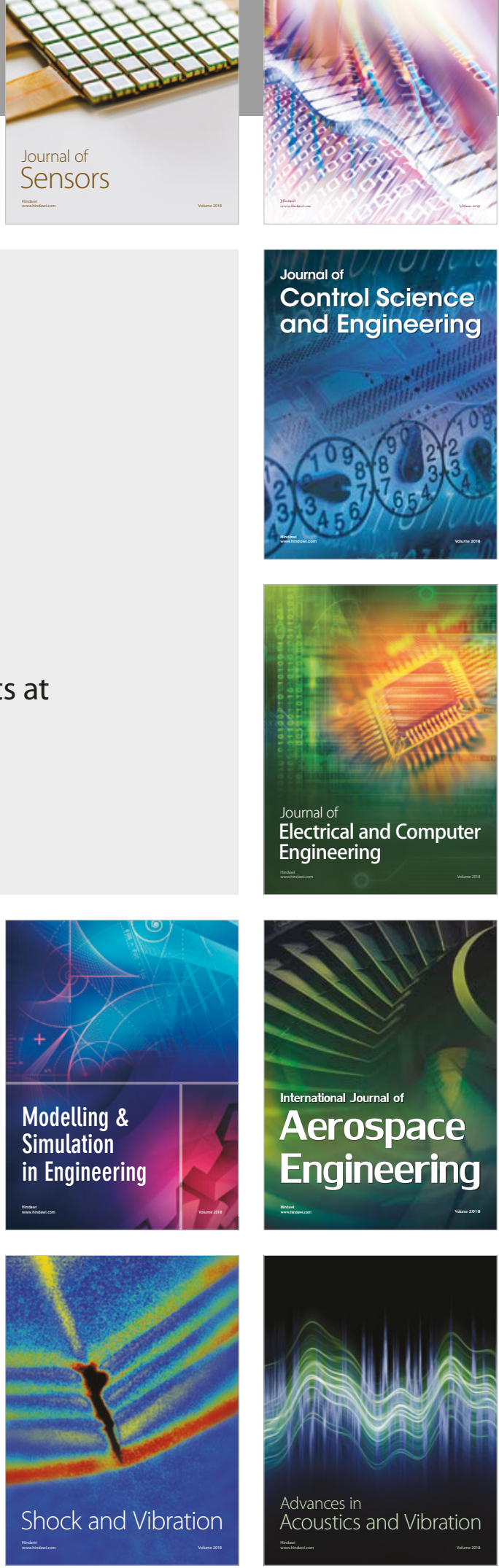